\title{
Distributed online RWA considering add/drop contention in the nodes for directionless and colorless ROADMs
}

\author{
P. Pavon-Marino, M. V. Bueno-Delgado \\ Universidad Politécnica de Cartagena (Spain). Pza. Hospital 1, 30202, Cartagena, Spain \\ E-mail: \{pablo.pavon, mvictoria.bueno\}@upct.es
}

\begin{abstract}
This paper presents an online distributed RWA algorithm that considers the add/drop ports occupation in directionless-colorless ROADMs, to reduce the lightpath blocking. Results support our approach to reduce the number of ROADM transponder banks. OCIS codes: (060.4251) Networks, assignment and routing algorithms ; (060.1155) All-optical networks
\end{abstract}

\section{Introduction}

In transparent optical networks, traffic is carried over all-optical connections, called lightpaths. A lightpath originates at a transponder in the source node, where it is said to be added. It occupies a wavelength channel in each traversed link, and terminates at a transponder in the end node, where it is said to be dropped. Add, drop and optical bypass of the lightpaths is implemented by Optical Add/Drop Multiplexers (OADMs). OADMs with capabilities that permit agile reconfiguration of the lightpaths without manual intervention are called ROADMs (Reconfigurable OADMs). Current generation of ROADMs have limited versatility: an on-site technician visit is still required to change the direction of an added or dropped lightpath, or to change its wavelength. The interest of service providers in introducing lightpath-on-demand services has driven the evolution to next generation of ROADMs, with directionless and colorless add/drop ports. Directionless feature means that add (drop) transponders can access to any outgoing (incoming) route, under the control of the ROADM management plane. Colorless feature adds the possibility to change the wavelength in an add/drop transponder without manual intervention.

Colorless-Directionless ROADMs with full interconnection between any add/drop port and any route, at any wavelength would have an enormous complexity. Several ROADM architectures trying to address this issue have been proposed [1]. The most common approach is arranging the transponders into transponder banks, which are shared among all the directions. Fig. 1 illustrates this in a broadcast-and-select ROADM with two transponder banks, and three directions (North, East, West). The client-side fiber cross-connect (C-FXC) decouples ROADM transponders from client line cards, sharing the transponders among potentially many clients of the lightpath-ondemand service, and providing 1:N transponder protection.

As shown in Fig. 1, any transponder can reach any route at any wavelength. Then, both the directionless and colorless operations are guaranteed. However, internal contention occurs: two transponders of the same bank cannot

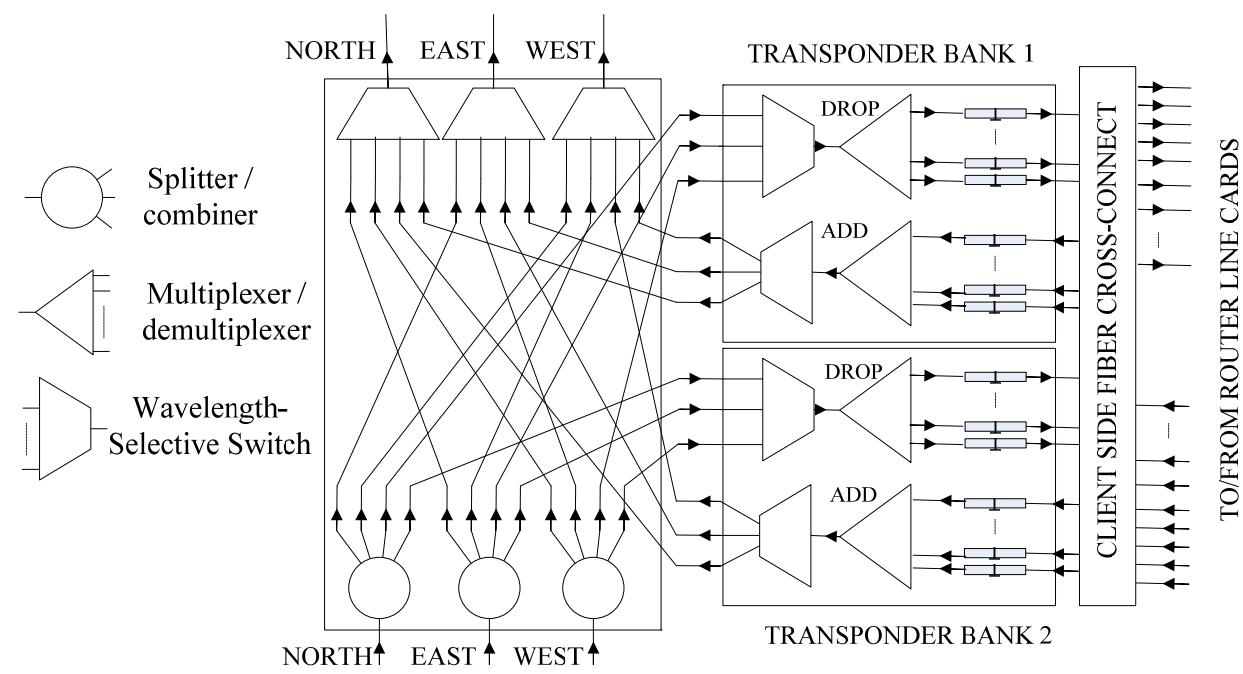

Figure 1: Broadcast-and-select colorless-directionless ROADM architecture with FXC at the client side 
be tuned at the same wavelength since only one fiber pair connects the transponder bank with the ROADM core. Naturally, the higher the number of transponder banks, the higher the cost and the smaller the lightpath blocking caused by the add/drop contention. This paper is targeted to study this cost vs. performance trade-off. In the limit, contentionless ROADMs are those for which the blocking caused by add/drop contention is eliminated in practice.

Add/drop contention-aware Routing and Wavelength Assignment (ADCA-RWA) problem has been investigated in a few works in the past [2-4], as a strategy to alleviate the blocking effects caused by internal contention. In [2] and [3] ADCA-RWA is addressed for offline planning, when all the lightpath requests are known in advance. [2] is focused on the unprotected and 1+1 lightpath protection cases, while [3] studies the lightpath restoration case. Results show that in these scenarios two transponder banks (and often just one) can be enough to achieve contentionless behavior in practice, if the RWA is adequately designed. The ADCA-RWA problem has been also investigated in the online planning scenario in [4], when lightpath demands arrive randomly to the nodes. In [4] several online ADCA-RWA planning algorithms are proposed and their performances are investigated. In the present paper, this approach is also followed, proposing for the first time an online ADCA-RWA algorithm that can be implemented in a distributed form and integrated in a standard GMPLS RSVP-TE network [5]. In addition, we include preliminary results using the algorithm to dimension the number of transponder banks required to eliminate add/drop contention effects, in a reference topology. They are the first results of this type, since the tests in [4] consider a number of banks always equal to the number of directions of the node. Results reveal that one or two transponder banks may be enough also in the online case to provide contentionless behavior in practice.

The rest of the paper is organized as follows. Section 2 presents the online ADCA-RWA algorithm, Section 3 the results obtained, and Section 4 concludes the paper.

\section{Online ADCA-RWA algorithm description}

We assume that a GMPLS control plane with RSVP-TE extension is running in the network [5]. Thanks to this, each node $n$ contains in its TE (traffic engineering) database updated information of the fiber topology, and can precompute a set of $k$ potential paths to each other node. The extra information the node handles is local: the lightpaths added and dropped, the occupation of the wavelengths in its own links, and the occupation of its own transponder banks. The actions taken when a lightpath demand arrives to node $s$, targeted to node $d$ are:

Step 1. Choose the shortest path route to $d$ in number of hops, among the precomputed paths not previously checked. If no route exists, the lightpath is blocked.

Step 2. Node $s$ sends a request to $d$ by means of a conventional RSVP Path message, to establish a lightpath in the selected route. In the label set object (LS) of the Path Message, include the wavelengths available in the first downstream link (according to [5]), but filter out from LS the wavelengths that are occupied in all the transponder banks in $s$, since they would suffer from add contention.

Step 3. According to the conventional GMPLS RSVP-TE protocol, the RSVP Path Message propagates in the same route as the one to be followed by the lightpath, and each node removes from the LS field the wavelengths that are already occupied in its downstream link.

Step 4. When the RSVP message arrives to $d$, the wavelength list is checked against the drop contention-free wavelengths in $d$. If valid wavelengths exist, select one using first-fit. If not, go to Step 1.

The algorithm description omits many RSVP-TE signaling details (i.e. the RSVP Resv message and backward reservation procedure), since they are entirely equal to [5]. In fact, the previous algorithm can be directly applied to GMPLS RSVP-TE [5] control planes, with no changes in the lightpath signaling procedures. This is acquired thanks to two simple actions: (i) in Step 2 node $s$ does not include in the LS field the wavelengths subject to add contention in $s$, and (ii) in Step 3, node $d$ does not choose a wavelength subject to drop contention in $d$. Note that after a contention-free wavelength-continuous RWA is selected, any transponder bank in $s$ and $d$ that is contention-free for that wavelength can be chosen (e.g. the one with more idle transponders). The algorithm guarantees that such bank exists in both $s$ and $d$.

\section{Results}

The proposed approach is evaluated by means of event-driven simulation using the MatPlanWDM platform [6]. In our tests, we consider the Internet 2 topology ( 9 nodes, 26 links) and the reference traffic matrix $M$ for this topology published in [7]. The number of wavelengths per fiber tested are $W=\{40,80\}$. Nodes are equipped with $C=\{1,2,3, \infty\}$ transponder banks. The case $C=\infty$ represents the contentionless case. The number of transponders in each bank is considered abundant. Lightpath requests arrive randomly to the nodes with exponential interarrival time distribution. Lightpath duration is also random, with an exponential distribution of average 1 time unit. The average 
load generated between each $(s, d)$ node pair is equal to $\rho M_{\max }(s, d)$. The factor $\rho$ is a network load normalization factor $(0 \leq \rho \leq 1)$. The matrix $M_{\max }$ is calculated as in [2]. It corresponds to the largest lightpath demand matrix proportional to $M$, that can be supported with null lightpath blocking by a network equipped with contentionless ROADMs, wavelength converters in the nodes, and for which the demand arrivals are not random. The simulation ends when at least $100 \cdot \mathrm{W}$ lightpaths are established between the node pair which have less lightpaths established between them. Ten runs are averaged in the results. In each run, transitory time is a $10 \%$ of the total simulation time.

For each lightpath demand, the algorithm is executed with parameter $k=10$. If valid RWA and contention-free transponder banks are found, the lightpath is allocated in the network accordingly. If not, the lightpath is blocked. Figs. 2(a-b) show the blocking probability as a function of the network load factor, for $C=\{1,2,3, \infty\}$ transponder banks. Comparing the curves $C=2$ with $C=\infty$ we spot that two transponder banks suffice in practice to have a contentionless behavior. Moreover, at loads $\rho \leq 60 \%$ (at $W=40$ ) and $\rho \leq 70 \%$ (at $W=80$ ), a network of ROADMs equipped with one transponder bank already provides a lightpath blocking below $0.1 \%$. The trend is that higher number of wavelengths results in lower lightpath blockings for the same load factor in all the cases. In the $W=80$ and $C=2$ tests, a lightpath blocking greater than $0.1 \%$ was only found in the $\rho=100 \%$ run.
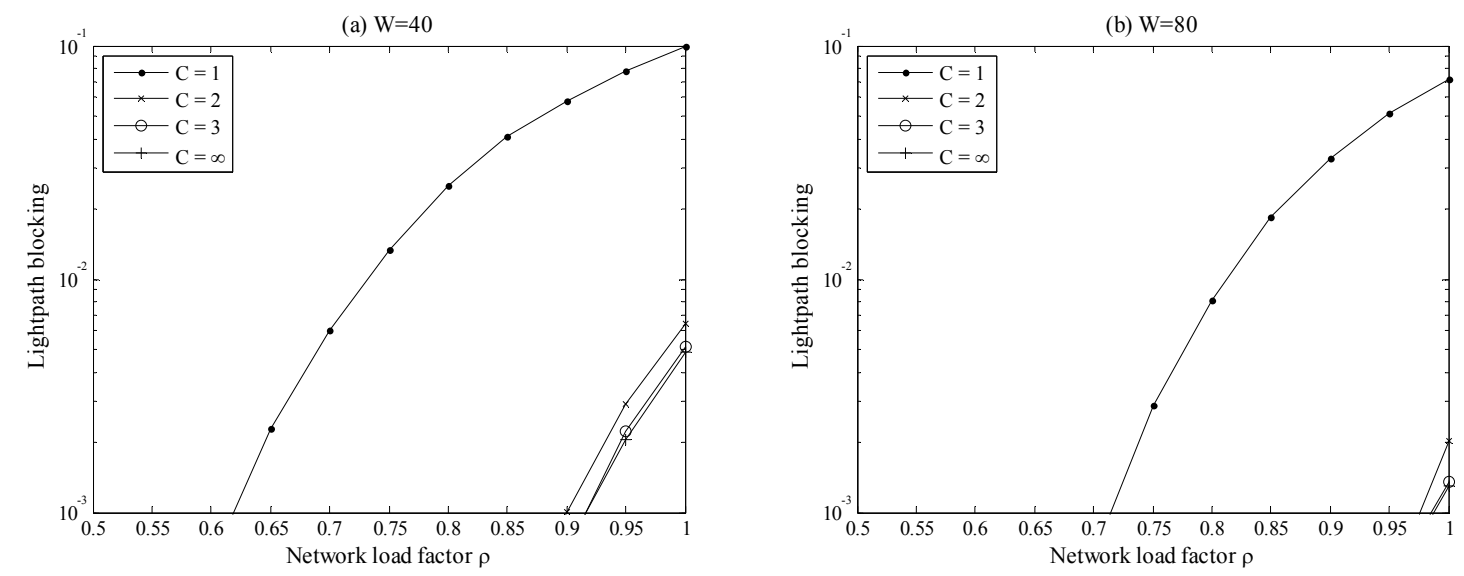

Figure 2: Lightpath blocking performance

\section{Conclusions}

This paper presents for the first time a distributed add/drop contention-aware RWA algorithm (ADCA-RWA), suitable for networks equipped with colorless-directionless ROADMs. The algorithm can be readily integrated in a GMPLS RSVP-TE control plane with no changes in the lightpath establishment procedures. We conduct a battery of tests using the Internet 2 reference topology. Results show that the algorithm is effective alleviating the add/drop contention effects. In our tests, two transponder banks were enough to provide a contentionless behavior in practice. One transponder bank was enough in low and medium loads (i.e. load below or equal to $70 \%$ in the $W=80$ wavelengths per fiber case). The authors are working in an exhaustive battery of tests, including different network topologies, to investigate the validity of these dimensioning rules in other scenarios.

\section{Acknowledgements}

This work was partially supported by Spanish projects grants TEC2010-21405-C02-02/TCM (CALM) and TEC2010-12250-E (FIERRO), and also developed in the framework of project "Programa de Ayudas a Grupos de Excelencia de la Región de Murcia” funded by F. Séneca (Plan Regional de Ciencia y Tecnología 2007/2010).

\section{References}

[1] S. Gringeri, B. Basch, V. Shukla, R. Egorov and T. J. Xia, "Flexible architectures for optical transport nodes and networks", IEEE Communications Magazine, vol. 48, no. 7, pp. 40-50, Aug. 2010.

[2] Pablo Pavon-Marino, M.V. Bueno-Delgado, "Dimensioning the add/drop contention factor of directionless ROADMs", to be published in IEEE/OSA Journal of Lightwave Technology.

[3] Pablo Pavon-Marino, M.V. Bueno-Delgado, "Add/drop contention and lightpath restoration performance in directionless ROADMs", Technical Report (1)03-2011-TIC-UPCT, submitted to IEEE/ACM Transactions on Networking.

[4] P. Palacharla, X. Wang, I. Kim, D. Bihon, M. D. Feuer, S. L. Woodward, "Blocking performance in dynamic optical networks based on colorless, non-directional ROADMs", in Proc. OFC/NFOEC 2011, paper JWA8.

[5] L. Berger (Ed.), “GMPLS Resource Reservation Protocol-Traffic Engineering (RSVP-TE) Extensions”, IETF RFC 3473, Jan. 2003.

[6] MatPlanWDM Web Site [Online], http://ait.upct.es/ ppavon/matplanwdm/.

[7] Internet 2 Global Research Network Operations Center, Web Site, [Online], Available: http://www.abilene.iu.edu/. 